\title{
New Insights of the Systematic Approach to Training (SAT): A Quality Governance Perspective
}

\author{
Dalia Mohamed Mostafa Mabrouk ${ }^{1,2}$ \\ ${ }^{1}$ Faculty of Arts \& Humanities, Suez Canal University, Ismailia, Egypt \\ ${ }^{2}$ Abu Dhabi Police General Headquarter, Abu Dhabi, UAE \\ Email: dmabrouk2009@hotmail.com
}

How to cite this paper: Mabrouk, D. M. M. (2021). New Insights of the Systematic Approach to Training (SAT): A Quality Governance Perspective. Open Journal of Social Sciences, 9, 425-437. https://doi.org/10.4236/jss.2021.91031

Received: December 21, 2020

Accepted: January 25, 2021

Published: January 28, 2021

Copyright (c) 2021 by author(s) and Scientific Research Publishing Inc. This work is licensed under the Creative Commons Attribution International License (CC BY 4.0).

http://creativecommons.org/licenses/by/4.0/ (c) (i) Open Access

\begin{abstract}
In this paper, I embark on diving deep into the specific training model of the systematic approach to training for probing more perspectives of its governance that should connect and link its phases together. Actually this training model is applied by many organizations all around the world that require a high level of professional trained staff with a minimal standard deviation of processes. I am here concerned with those organizations seeking a better training system, while ending up being superficially satisfied with reverberating and bragging with having the SAT onboard without full awareness of the possibilities of its implementation. It is not about the system, it is about the mindsets that are flexible enough to fully grasp the ins and outs of the model they are about to use and how to harness its tools for their organizational benefit; otherwise it's a waste of time, effort and money. In order to operate training in a systematic manner, it has to not only cover the inter related stages and processes, but also set a solid bridge that keeps and maintains the smooth landing from one stage to another along with securing the prospected outputs which conforms to both form and content. Based on my experience on the best practices of the systematic approach to training, I will here contribute my insight of the system quality control technique that may help in a well-knitted flow of processes.
\end{abstract}

\section{Keywords}

Systematic Approach to Training, SAT, Development, Training, Learning Styles, Evaluation of Training

\section{Introduction}

In this paper, I am concerned with finding answers to certain enquiries about the 
SAT system; could the development phase be combined with the design phase without affecting the prospected outcomes for both? Is the evaluation phase enough for ensuring the competency of the system? Or another methodology has to be added for maintaining quality and saving time exerted for later discovery of pitfalls through the evaluation process?

Let's start with the classic approach to define what training means? Training has been defined as "The systematic development of the knowledge, skills and attitudes required by an individual to perform adequately a given task or job" (Ribbler, 1983). Training has also been defined in the Glossary of Training Terms (Manpower Services Commission, UK) as "a planned process to modify attitude, knowledge or skill behavior through learning experience to achieve effective performance in an activity or range of activities. Its purpose in the work place is to develop the abilities of the individual and to satisfy the current and future manpower needs of the organization". It clearly implies that the role of training is to improve the overall performance of the organization. The term "performance" is, therefore, interwoven with training.

Organizations who aspire to achieve their strategic goals of improving performance should adopt proper training methodology which enhances the professional knowledge and skills both at individual and collective levels. (Goldstein, 1986). On top of that, knowing how to function and integrate the whole phases is as well of a paramount importance. It should also equip personnel with proper skills to respond appropriately to the emerging challenges in the inner and outer environment as the COVID-19 pandemic. Training should also bring about appropriate changes in attitudes and should strive for that unique synthesis between improvement of the individual's competencies and fulfilment of organizational objectives.

Moving to the systematic approach to training as one of the most common training models well known for its peculiar qualities of qualifying personnel in a professional and practical way, I am interested to dive deep into its stretching fabric of implementation possibilities. The US Department of Defense (Marine Corps) in its Systematic Training Manual designs the procedures for developing training curricula and managing the training unit of workforce. Therefore, their manual was being developed by the Ministry of Defense in order to be the primary source of information on which the Navy and other military service schools and workforce units rely. The systematic approach to training model is built on Instructional System Development (ISD), which is characterized by flexibility in order to effectively fit into the institutional structure. For governance purposes, decisions regarding training programs are taken by the (Central Training Unit) of the head Office of the Commander in Chief (SAT Manual, 2004).

Reasons for implementing the Systematic approach to training model (SAT):

Though this model has been conducted in the early 70ths, it is noteworthy that the US military operations that took place in Iraq and Afghanistan prompted the military leadership to develop its training curricula to enable the Marines 
to better deal with the challenges of the field reality they found themselves dealing with. In order to achieve this, the approach was developed based on the development of education and training processes, which produced the (ISD) model, later known as the (SAT) model.

The mission of any training system is to define the educational needs and priorities, develop effective and efficient solutions to meet these needs, and implement these solutions in a practical manner (Albela, 1986). To achieve this, teaching and training methods were developed. Therefore, the US Ministry of Defense has been diligently working to develop the SAT manual to be more comprehensive for procedures that accommodate and encourage the cognitive and emotional development of the Marine Corps in particular, and all sectors of the Ministry of Defense in general.This model, whether referred to as ISD or SAT, is a recognized standard that governs the educational and training process within the Department of Defense (DoD) and other US government institutions.

Training objectives of the US Department of Defense were mainly based on enhancing the trainees' capabilities and competencies in their transition from the training field to the real job performance. As a result, they have to ensure that learning objectives are effective and applicable, placed on the basis of job functions and the utmost utilization of resources.

It is also worth mentioning that the SAT model is comprehensive and flexible which defines what the tasks and responsibilities of the job are, what training requirements are and how they should be designed, implemented and evaluated? It ensures that what are being trained on are the most important tasks for a successful job performance. It also ensures that the educational methods and media are the most time- and cost-effective possible (Davis \& Davis, 1999). It also confirms that the trainees are evaluated on the extent to which learning objectives are achieved in a way that allows improvements and reviews to be conducted for measuring the effectiveness and efficiency of training programs.

What distinguishes the systematic approach to training SAT is eliminating the decision-making process which is based on personal opinions and paving the way for decisions to be made based on logical conclusions due to careful data collection and statistical analysis (Bass \& Vaughan, 1966). Then determining whether training is the best solution for developing job performance or other methodologies.

Though I am not concerned here with giving a full detailed explanation of the phases of the SAT system, I will go over the various stages and how they are integrated to fulfill the final goal of the organization and to bridge the gap between the aimed performance and the actual one (See Figure 1). The decision makers and the persons in charge of training should have this panoramic scope of vision of how the systematic approach to training should work in order to fit their own organization's purposes. For instance the various phases of the system could be handed over to different sectors or departments within the organization, still they should recognize the importance of collaboration and co-ordination with 


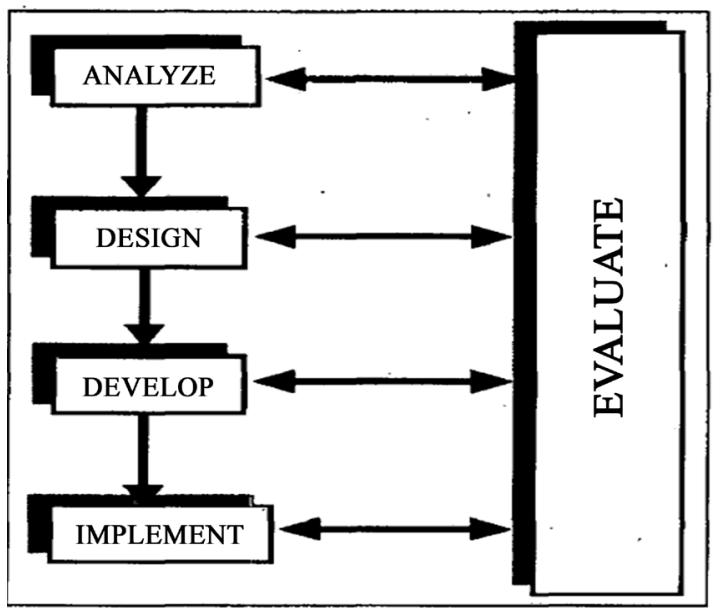

Figure 1. The systematic approach to training as applied in the US Marine Corps. It consists of 5 integrated phases; analysis, design, develop, implement and evaluation.

each other; not working as isolated islands. Another point I come across as firsthand experience with the implementation of this system is that the whole focus and concentration of those working on the phases should be focused on releasing the best version of the course portfolio, for example the mission of the analysis team doesn't end with handing the job analysis portfolio to the successive phase (the design phase), but they are also expected to contribute their hints, suggestions and modifications to the master Learning file (MLF). Again the design and the development phases should have as well their contributions to modify the MLF. It is not exclusive to a certain phase or certain personnel. It is mainly why the system works; for releasing the best version of the training course content before the training delivery starts.

\section{Discussion}

The SAT model normally starts with the analysis phase. This stage defines the needs and requirements of the job/task; it examines the gap between the desired performance and actual performance. In the analysis stage, the ultimate goal is to find out if training is required as a solution to a performance problem. The analysis process is carried out on several levels :Job analysis, task analysis and training needs analysis. (It is to convert all outputs of the analysis process into a systematic file to conduct a training course.)

\section{Analysis phase}

Job analysis is a systematic process for defining and detailing the duties and tasks of a particular job and mentioning its requirements and importance for the rest of the jobs in the organizational structure, and determining the job level in terms of activities, tools for performing those activities and the circumstances in which the job is being performed. It is considered the preparatory stage for writing a job description, and it includes a precise description of the job characteristics that must be met by certain qualifications, and presenting the mental and physical requirements accompanying work conditions (Swanson, 2001). 
During this process, the importance of the job is judged through data collection, with an emphasis on the fact that the judgment is made on the "job" and not on the "person" occupying the job. This is one of the important concepts that are focused on while working on data collection for the purpose of analysis (Laird, 1985). There are several analytical methodologies used to collect and analyze data, including: interviews, questionnaires, or the observation method. The final result of the job analysis process is to provide a comprehensive analysis and description of the job compared to the organizations' peers or its counterparts in other bodies.

The purpose of the job analysis is to create a job description and define performance requirements upon selection and appointment of candidates in terms of capabilities, skills, experiences, characteristics, and technical knowledge, and to define the training needs for occupying the position and also define the performance evaluation criteria (Rothwell, 1996). Russian psychologist Maurice Felts was the first to use job analysis in 1922 to select employees for Trolley Cars. As the aim of the analysis process is to identify performance requirements and deficiencies, therefore it must answer 4 basic questions:

1) What are the tasks that employees actually do?

2) What are the tasks that should be performed by the employees?

3)What is the reason for the gap, if any, between actual performance and the required performance?

4) Will training be part of the solution?

\section{Design phase}

The second phase of the SAT model is (the design.) Its processes are managed for forming the basic foundation of the training material. The output of the analysis stage is considered the main input of the design phase. On top of this, determining whether the best option is to conduct training with clarification of the features of the appropriate training environment to meet the needs such as training in laboratories or simulation exercises or Standard classroom training are all about to be customized in a course portfolio (Smith \& Ragan, 1999).

\section{Development phase}

Then the training performance statement is used to compile the elements and contents of the training course plan, define the trainer's tasks and the trainees' activities. Then comes the development phase to develop the supporting materials for the training program and make sure that all the training aids are available and provide the suitable alternative for any missing teaching tools or equipment (Bloom, 1956). There are certain organizations who would combine both the design and development in one phase. Such step is little risky as it requires the pertinent training management to set a well-knitted quality control plan over the mechanism of processes to assure smooth release of totally completed course file and full preparation of training set.

As mentioned before, the design phase is based on analyzing the job file to design the general structure of training inputs, where all data is converted into les- 
son plans and training materials as a reference for trainers and ready to meet the needs of job tasks and improve the performance required to be achieved (Tracey, 1974). It is where material experts and program designers participate in preparing suitable training materials for achieving the desired training objectives.

Normally, the design stage has results in the production of the description of the training program (lesson plans and needed training resources), the requirements of the trainee's enrollment level in the course, review of learning objectives, the gradual sequence of objectives and identification of educational methods, media and resources (SAT Manual, 2004).

\section{Conduct phase}

Then the conduct or implementation is the fourth stage of the Systematic Approach to Training model, in which the training programs are put into practice, which is the culmination of systematic planning and development in which the trainees derive the benefit of previous efforts through effective training. This stage is managed by a qualified, experienced and skilled training staff in order to deliver the training course.There are 4 main activities in the implementation phase; preparing a training plan, course delivery, measuring the effectiveness of training implementation and documenting the training processes (SAT Manual, 2004).

Among the most important outputs of training implementation processes (trained staff/attendance records/internal evaluation report/trainers qualification documents/individual training records.) The implementation phase employs the outputs of the design and development phases to conduct an actual delivery on an experimental course.

\section{Evaluation phase}

The outputs of the implementation phase are:assessing the training plan, performing a trial course experiment, conducting an evaluation of the training program experience and documenting training implementation processes. In this stage, the effectiveness of the training is evaluated and opportunities for continuous improvement are identified.This stage is based on ensuring that the outputs of the training processes produce competent employees and achieve the desired learning objectives (Mager, 1984). Here, the evaluation and monitoring of training programs are carried out and modified as necessary. The program evaluation process mainly includes the results of the training program and its effectiveness.

This stage depends on three main activities; follow up on monitoring indicators, analyze the information and initiate corrective actions. While the follow-up phase includes the following indicators; variety of training methods used, reports of supervisor and administrator, results of examination and evaluation of training operations and any modifications or changes to the training process. The evaluation stage is the last stage of the systematic training system, whereby supervisors, trainers and administrators are constantly monitored for the purpose of evaluation during training operations. This allows them to adjust methods as 
necessary during the sessions. In addition, there is also a stage to collect trainees' feedback after completion of the training course. The evaluation stage then collects comments and opinions and uses this information to redesign the lesson plan as well as the job file. The concerned authority can follow up on the level of development in the performance of the trainees after their return to work to collect more evaluation information, as the evaluation stage collects feedback and uses that information to redesign the lesson plan.

The outputs of the evaluation stage are; internal evaluation report, external evaluation report, analyzing information about the program's training process, proposed corrective actions, regulatory developments and evaluation data during training (trainers and trainees). It is a process that confirms the effectiveness of training and development in the organization, cost-appropriateness and equipment, and is grouped based on internal and external evaluation reports.

Furthermore, it would be feasible to monitor and assess the following; monitoring indicators, information analysis, corrective actions, planned courses for the systematic training system and the responsibilities and tasks of those involved in training operations. So it is obvious that the SAT model has certain peculiarities than any other training model. It sets the term "training" simply beyond organizing classroom sessions. There are more to plan for. This process enables stakeholders to identify the role of the personnel responsible for organizing training and implementing the training policy. Consequently, after following through all these steps with full awareness of the connectivity among the various phases, the concerns of the overall governance of the system could be figured out: do we need to do the verification of the whole system in the same phase of evaluation? Or it would be better to separate it in a specific phase as part of the SAT lifecycle itself? What answers these questions is the practical application of the system and what inner procedures are most suitable for the organization itself. The SAT model is a very competent system for conducting a training course according to the specified needs of the organization if implemented according to the stipulated requirements. Then a methodology has to be put in place for monitoring the smooth operation of the internal processes of the phases as well as the integration of the outputs before handed over to subsequent phase.

According to the US Department of defense model of the SAT system, they set a methodology of evaluating the whole system through the evaluation phase. When there is a direction of conducting a certain course, the TEC committee assigns the specialized team to commence the evaluation process through a pre-set agenda. They may start with checking the detailed plan of the analysis team which is standardized as (planning/conduct/data analysis/data management/results and modifications on the Master Learning Portfolio) (see Figure 2). The same process also applies to the design and development phases. Each phase has to impact and contribute to the MLF with the purpose of reaching to the most suitable version of course content that would achieve the learning objectives as well as the organization's performance indicators. When it comes to verification the processes 


\section{Stages of the evaluation process}

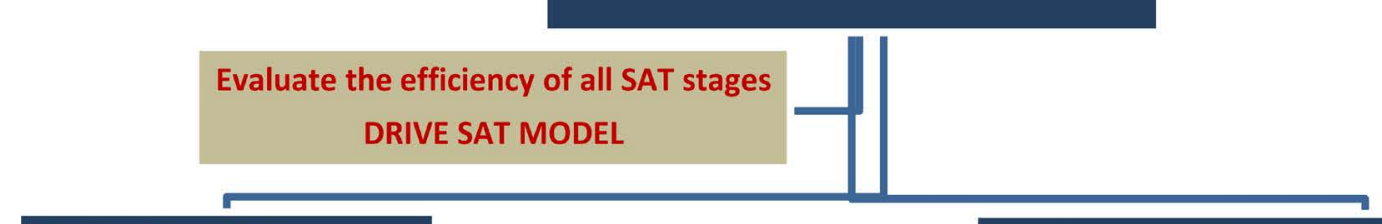

\section{Evaluation of the system}

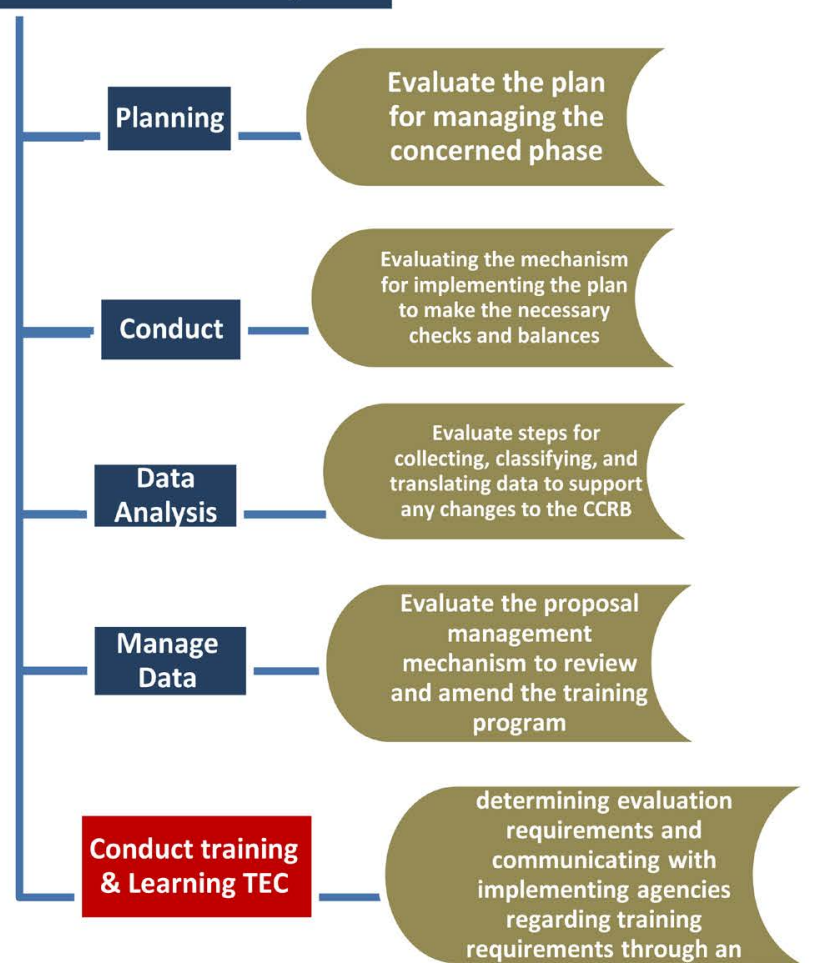

Evaluation of training conduct

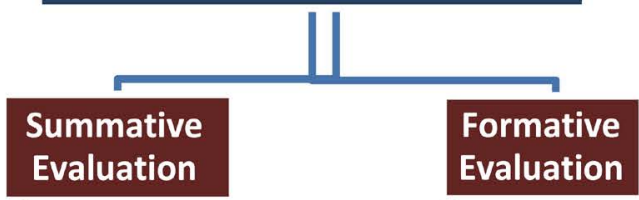

Reviewing and

evaluating the

performance of

trainees, trainers,

training materials

and the training

environment after

the start of

implementing the training

\section{Evaluation}

Figure 2. Shown how the evaluation phase is managed through a systematic process to the whole SAT stages.

of training validation, things here have a different scenario. There is a specified team (TEC) that works as the centralized committee for conducting and driving the verification teams. They have the authority to assign teams for targeting the phases for verification at any time, and it's not necessarily to start verification of all phases for every training course. In other words, if the TEC needs to check whether the trainees grasp the learning objectives, they would assign the team for verification of the implementation phase, or if they need to check whether the learning points completely covered the job tasks, they would send the teams for verification of the design process.

Due to the maximum importance of the Master Learning File (MLF), it needs to pass through many filters for modifications and refinement before handed over to the trainer. This justifies the necessity of verification of all phases, and not only focuses on the internal and external validation. MLF includes Contents of the course plan file or the documents that are required to be reviewed when conducting an evaluation of any stage of the SAT system, which is mainly related to the course and the point of implementation (see Figure 3). 
Training Manual / Standards for Individual Training

\section{Program description CDD /} And POI tutorial

\section{MLF master lesson plans file}

- The basic rule for building instructions and policies

- Identify any gaps between program content and educational objectives

- It provides essential data for evaluating the course program

- Includes (resources needed / learning objectives / training hours / number of trainers / methods and

media ...)

- Essential for comparing the outputs of the training process with the contents of the file and monitorin the feedback from any observations or errors (for existing courses). If there are no errors in the lesson plans file, the problem lies in the lack of compliance and adherence to the file by the trainer

- Defines the mechanism for gathering and analyzing data and the resources available to provide the assessment data. It is the basic basis for carrying out evaluations of the design / development / implementation / evaluation phase.
Manual of operational procedures for implementing the SOP training

\section{Evaluation plan}

- The evaluation plan should be revised to match the evaluation processes in accordance with the approved policies. The evaluation plan can be included in the operating manual

\section{Audit Reports / Visiting reports}

\section{ROP Procedure Log}

- Some specialist units have inspection teams to ensure quality. The real challenge lies in determining whether the discovered strengths and weaknesses are due to the training implementation body or the trainee's organizational unit.

Figure 3. Shows the contents of the master learning file (MLF) that should be reviewed carefully through the evaluation processes.

Blueprint of the contents of the master learning file (MLF):

All organizations willing to adopt the systematic approach to training model should secure proper procedures of answering certain questions about each phase to maintain the effectiveness and efficiency of the whole system. Certain points to be taken into account; What is the necessary evaluation mechanism to discover a gap/or inconsistency in the content of the program in terms of the learning objectives? What is the mechanism for ensuring the validity, comprehensiveness and suitability of learning objectives for targeting weaknesses related to job tasks? (Bienvenu, 1969).

The criteria of evaluating the various phases of the systematic approach to training:

Actually, the evaluation of the whole system has to be clarified through the action plan as mentioned before. Here are the questions to be taken into account; what is the necessary evaluation mechanism to discover a gap/or inconsistency in the content of the program with the approved educational objectives? What is the mechanism for ensuring the validity, comprehensiveness and suitability of educational objectives for targeting weaknesses related to job tasks? The criteria of the evaluation on the stages of the systematic training system are fully 
Evaluation of Design Phase

1) Do the knowledge and skills accurately reflect what needs to be trained for each task?

2) Do the educational objectives support the training needs of the target group?

3) Do the educational objectives accurately define the knowledge and skills needed to perform the job?

4) Do the tests measure the knowledge and skills that have been trained on?

5) Is the test method appropriate to the nature of the material (practical / theoretical) ?

6) Do the elements and levels of evaluation (tests) explain the difference between an efficient and incompetent trainee?

7) Is the type of training identified appropriate to enable the trainees to acquire knowledge and skills?

8) Are the educational points arranged in a logical order to raise the level of understanding of the lesson plans?

Evaluation of the implementation phase

Course evaluation

1) Do the educational materials support the learning objectives?

2) Are the training aids suitable for teaching and the topic?

3) Are the test instructions clear and understandable?

4) Do students have all the materials (equipment, calculator, etc.) necessary to complete the test?

5) Do students use the course materials available to them? Trainers' evaluation

1) Does the trainer encourage the participation of trainees?

2) Does the trainer provide feedback to the trainees?

3) Does the trainer have sufficient knowledge of the course materials?

4) Does the trainer communicate and interact effectively?

5) Does the trainer use the media effectively?

6) Is the test administration effective?

Assessment of the educational environment

1) Does the educational environment support the maximum level of understanding and learning?

2) Do the resources available allow the training course to be a list of the best forms of practical performance?

3)Is the trainer / student ratio appropriate?

Evaluate the trainees' behavioral change

1) Do the trainees master the learning objectives?

2) Are the trainees able to perform the tasks?

3) Do the trainees frequently encounter any problems in carrying out tasks or tests?

Figure 4. Explains the points that should be covered in the evaluation processes of the whole phases.

described in details above (see Figure 4).

\section{Conclusion}

Organizations whether governmental, public or private have to transform the mechanics of employee training especially after the world pandemic of COVID-

19. It is good to seek well-structured and quality driven training models as the system approach to training, but they have to be attentive of the methods and tools utilized for achieving their strategic goals. Copy and paste training models from other institutions without grasping the inner needs and enablers would complicate matters. That's why the upper management has a main role in supervising and monitoring the implementation as well as following up the recom- 
mendations of technical reports. Employees, on the other hand, have to take more responsibility in their professional journey of self-learning. They have to stop waiting for their organizations to spoon feed them of the training skills needed, and start take initiatives to join e-courses they consider of importance to update their work competencies.

Moreover, I would like to focus on the fact that the systematic approach to training (SAT) is not an end in itself; it's just a means for solving a frequent organizational problem such as low customer satisfaction or unplanned absences of employees or decreased productivity. Having the American model of the Systematic Approach to training under scrutiny, we may get out with several insights. Though this SAT model is very flexible and customizable, still without a clear strategy of governance to control and assess the outputs of each phase, organizations are wasting their money and efforts in vain. Another point needs to be considered is that the evaluation process which is mainly considered to fall into two categories; formative and summative is completely different from assessing the competency and effectiveness of the whole system. In other words, while focusing through the entire evaluation phase on measuring the training process only from (trainees/trainers' impression/job behavior/institutional impact), we should bear in mind that any deficiency in the analysis or design or development phases may drastically affect the training outcomes in general (Seels, 1998). So the absence of a strategic plan to assess the stages of analysis, design and development would consequently lead to a weak ability to determine the basis of training problems and thus waste of time and resources as well as inability to make the necessary corrections.

Formative evaluation is concerned with evaluating the educational content and lesson plans based on the training performance statement and analyzing needs before the implementation of the course in order to make any necessary amendments to the course plan file. While the summative evaluation is generally conducted to take the most appropriate decision regarding the course whether it needs development, suspension or continuation. Specialists handle evaluating the compliance and effectiveness of training delivery according to the prescribed procedures which could be managed by internal or external body to measure the efficiency and effectiveness of the course as a whole, including the performance of trainers, trainees and the educational environment.

Regarding the application of Kirkpatrick methodology of evaluating the training process and its effectiveness is not binding when it comes to the application of the systematic training system (SAT). Any organization willing to adopt the SAT model is free to employ the mechanism of evaluation that best achieve the prospected results as well as measure the efficiency of the system as a whole. So it is recommendable to review and study the best practices implemented by other organizations in terms of the evaluation processes. For example it is applied at The International Atomic Energy Agency (IAEA) as well as the Nuclear Energy Center in Cyprus as part of the systematic training system. They prefer to clearly 
cover the four areas of Kirkpatrick evaluation model (trainees' impression, learning accomplishment, behavioral change and organizational impact) (Kirkpatrick, 1993). We couldn't go for or against it as long as it helps achieving the final results. Still it seems plausible to adopt any model that secures and guarantees the data base for evaluating the course delivery and correcting deviations in a well-structured and well-knitted manner.

To conclude I have to highlight the importance of the upper management commitment to supervise and put the systematic approach to training model and its proper implementation as a priority on its agenda. Management commitment here means recording their observation and giving purposeful feedback on the raised reports for establishing a record trail that would pave the way for giving right decisions on training courses and its final cost effectiveness. On top of that having the intellectual openness and knowledgeable mindset to grasp the model as flexible enough to be tailored for fitting the needs and the requirements of the organization would definitely smooth the application procedures. So the success of the SAT system doesn't depend on one factor, but on a set of three main factors: management involvement, qualified personnel and compliance with the approved procedures.

\section{Conflicts of Interest}

The author declares no conflicts of interest regarding the publication of this paper.

\section{References}

Albela, K. T. (1986). Building Successful Training Program. Reading, MA: Addison-Wesley Publishing Inc.

Bass, B. M., \& Vaughan, J. A. (1966). Training in Industry: The Management of Learning. Belmont, CA: Wadsworth.

Bienvenu, B. J. (1969). New Priorities in Training-A Guide for Industry. Academy of Management Journal, 12, 527. https://doi.org/10.5465/amj.1969.19201259

Bloom, B. S. (Ed.) (1956). Taxonomy of Educational Objectives. New York: Longman.

Davis, J. R., \& Davis, A. B. (1999). Effective Training Strategies: A Comprehensive Guide to Maximizing Learning in Organizations. San Francisco, CA: Berrett Koehler.

Glossary of Training Terms (1981). Training Services, Manpower Services Commission: H.M.S.O. (3rd ed.). National Government Publication.

Goldstein, I. (1986). Training in Organizations: Needs Assessment, Development \& Evaluation. Monterey, CA: Brooks Cole.

Kirkpatrick, D. L. (1993). How to Train and Develop Supervisors. New York: AMACOM.

Laird, D. (1985). Approaches to Training and Development. Reading, MA: Addison Wesley Publishing Co. Inc.

Mager, R. F. (1984). Preparing Instructional Objectives (2nd ed.). Belmont, CA: David S. Lake Publishers.

Ribbler, I. R. (1983). Training and Development Guide. Reston, VA: Reston Publishing Co. Inc. 
Rothwell, W. J. (1996). Beyond Training and Development: State of the Art Strategies for Enhancing Human Performance. New York: AMCOM.

Seels, B. (1998). Making Instructional Design Decisions. Merrill Education/Prentice Hall.

Smith, P. L., \& Ragan, T. J. (1999). Instructional Design (2nd ed.). New York: John Wiley \& Sons, Inc.

Swanson, R. A. (2001). Human Resource Development and Its Underlying Theory. $\mathrm{Hu}$ man resource Development International, 4, 299-312. https://doi.org/10.1080/13678860110059311

Systems Approach to Training (SAT) Manual (2004). United States Marine Corps, Marine Corps Combat Development Command, Quantico, Virginia 22134-5001.

Tracey, W. R. (1974). Designing Training and Development. New York: AMCOM. 